\title{
LA EMPRESA EDUCATIVA CHILENA
}

\author{
JENNY ASSAÉL BUDNIK* \\ Rodrigo CoRnejo Chávez ${ }^{*}$ \\ JUAN GONZÁLEZ LÓPEZ* \\ JESÚS REDONDO Rojo* \\ RODRIGO SÁNCHEZ EDMONSON* \\ MARIO SOBARZO MORALES ${ }^{* *}$
}

\begin{abstract}
RESUMEN: Este artículo describe y analiza las características del llamado "experimento educativo chileno", tanto en sus aspectos estructurales, como de implementación, así como sus esenciales consecuencias. Finalmente se describen las principales tendencias generadas desde los movimientos sociales, que han implicado procesos de resistencia a las medidas neoliberales, así como una incipiente capacidad de propuesta alternativa. Para ello se revisaron un conjunto de fuentes primarias y secundarias de investigación sobre los alcances de este modelo. El artículo muestra que la aplicación sistemática de políticas educativas neoliberales es algo inédito en América Latina y que ha generado fenómenos y procesos sociales muy particulares, tanto de reproducción del modelo, como de resistencia social al mismo.
\end{abstract}

Palabras clave: Experimento educativo de mercado. Sistema educativo chileno. Movimiento social por la educación en Chile. Estandarización. Rendición de cuentas.

\section{The Chilean education enterprise}

ABSTRACT: This paper describes and analyzes the features of the so called "Chilean Education Experiment". It tackles its structural aspects, its implementation and its main consequences. It finally describes the trends that emerged from the social movements that have led to resist to the neoliberal measures and an incipient capacity for alternative proposals. It thus reviews a set of primary and secondary research sources on the outreach of this model. The text shows that the systematic application of neoliberal education policies is something

* Los autores son profesores del Departamento de Psicología, Facultad de Ciencias Sociales de la Universidad de Chile. E-mails: jennyassael@gmail.com (J.A. Budnik); rodrigo.cornejo@ u.uchile.cl (R.C. Chávez); juangl@uchile.cl (J.G. López); jredondo@u.uchile.c (J.R. Rojo); rosanche@uchile.cl (R.S. Edmonson).

** Departamento de Estudios Pedagógicos, Facultad de Filosofía y Humanidades de la Universidad de Chile. E-mail: mariosobarzo@gmail.com 
unheard of in Latin America that has produced very peculiar social phenomena and processes that either reproduce this model or socially resist to it.

Key words: Market education experiment. Chilean education system. Social movement for education in Chile. Standardization. Accountability.

\section{L'ENTREPRISE ÉDUCATIVE CHILIENNE}

$R E ́ S U M E ́:$ Cet article décrit et analyse les caractéristiques de la dite "expérience éducative chilienne", aussi bien dans ses aspects structuraux que dans sa mise en œuvre, et dans ses conséquences essentielles. Il expose ensuite les principales tendances engendrées par les mouvements sociaux, qui ont impliqué des processus de résistance contre les mesures néolibérales et une capacité balbutiante à faire des propositions alternatives. Pour ce faire, toute une série de sources primaires et secondaires de recherche sur la portée de ce modèle seront révisées. Le texte montre que l'application systématique de politiques éducatives néolibérales est quelque chose d'inédit en Amérique Latine et qu'elle a généré des phénomènes et processus sociaux très particuliers aussi bien de reproduction du modèle que de résistance sociale contre lui.

Mots-clés: Expérience éducative de marché. Système éducatif chilien. Mouvement social pour l'éducation au Chili. Standardisation. Reddition de comptes.

\section{Introducción}

$\mathrm{L}$

as políticas educativas implementadas en Chile, en las últimas tres décadas, han llevado, sistemáticamente, al desmantelamiento de la educación pública.

El sistema educativo chileno hoy se encuentra mercantilizado en su conjunto. La educación, de ser un derecho, se ha transformado en una mercancía, quedando a merced de enormes intereses empresariales. Lo paradójico es que este modelo se ha exportado al resto de nuestros países hermanos sin cuestionamiento alguno. Tal vez sólo las movilizaciones y protestas de los estudiantes secundarios, que tuvieron su momento culminante el año 2006 y que se conocieron como "revolución pingüina", trascendiendo las fronteras de nuestro país, levantaron la primera alerta respecto a sus carencias y debilidades.

Distintos autores han calificado el modelo chileno de "experimental", pues se implementaron (en plena dictadura militar) sin contar con evidencia empírica alguna que las avalara, un conjunto de políticas basadas en los postulados neoconservadores de economistas como Friedrich Hayek o Milton Friedman. Retornados los gobiernos civiles, a comienzos de los años noventa, se le dio continuidad a estas políticas; no deja de ser paradójico que incluso un panel de expertos de la Organización para la Cooperación y el Desarrollo Económicos (OCDE) concluyera que "la educación chilena está influenciada por una ideología que da una importancia indebida a los mecanismos de mercado para mejorar la enseñanza y el aprendizaje" (OCDE, 2004, p. 290-291). 
En este trabajo revisamos el proceso de implementación de este "experimento de mercado educativo", destacando los principales momentos de su instalación, los pilares en los cuales se sustenta y las consecuencias centrales que ha tenido su aplicación para el conjunto de los actores de la educación (estudiantes, docentes, familias).

Posteriormente, analizamos los aspectos esenciales de los heterogéneos, pero constantes procesos de participación de los actores sociales en el debate y la lucha educativa chilena. Esta participación tiene raíces históricas y durante los últimos años se ha sustentado en diversas iniciativas de resistencia y protesta frente a la imposición del modelo educativo neoliberal. Sin embargo, comienza a mostrar en su interior incipientes capacidades para generar propuestas educativas y experiencias de educación alternativas.

Construimos este artículo colectivamente desde nuestra experiencia como Observatorio Chileno de Políticas Educativas (оресн), investigando los efectos reales del modelo educativo de mercado y construyendo conocimiento en el contacto cotidiano con organizaciones sociales y educativas.

\section{Instalación del modelo chileno}

\section{La fase de shock en la década del ‘80}

En el proceso de constitución del Estado chileno, fueron explícitos la preocupación y esfuerzo por incorporar la educación como un derecho, y desarrollarla como una institución pública de carácter nacional. Esta responsabilidad del Estado, asumida por todos los gobiernos democráticos, aún de muy distinto signo, fue brutalmente alterada por el golpe militar de septiembre de 1973, que dio inicio a 17 años de dictadura, durante los cuales, a partir de la Constitución impuesta en 1980, se transforma profundamente el rol del Estado, de garante a subsidiario.

Hasta 1980, desde la concepción de un Estado docente que asegurara el derecho a la educación, existió en Chile un sistema público gratuito bajo la responsabilidad de un Ministerio de Educación fuerte y centralizado, que alcanzaba alrededor de un $70 \%$ de cobertura de la población en edad escolar y que atendía a más del $90 \%$ de la matrícula. Al comenzar la década de los $80^{\prime}$, la dictadura militar de Pinochet, en un contexto de represión que imposibilitaba cualquier tipo de cuestionamiento o debate, realizó cuatro grandes transformaciones que pueden ser consideradas los pilares del actual modelo. Éstas fueron, esencialmente: 1) redefinición del marco regulatorio educativo, del rol del Estado y de la prelación de derechos educativos a través de una nueva Constitución; 2) instalación de un modelo de gestión del sistema educativo formal que introduce un nuevo y central actor, los sostenedores (municipios y agentes privados); 3) implementación de una nueva lógica de financiamiento 
de los establecimientos educacionales, a través de una particular versión de vouchers o subsidios portables a los alumnos; 4 ) reestructuración y privatización del sistema de educación superior.

Todas estas transformaciones terminan por institucionalizarse a través de la Ley Orgánica Constitucional de Educación (LOCE), dictada el 10 de marzo de 1990, un día antes que Pinochet entregara el poder. Ninguna reforma de los gobiernos civiles de post dictadura han afectado estos pilares estructurales.

\section{Redefinición del rol del Estado: nueva articulación de derechos y nuevo marco regulatorio general}

La actual Constitución chilena fue promulgada en el año 1980 (en plena dictadura) y ha sido reformada sucesivamente sólo en aspectos marginales durante los gobiernos civiles. El apartado sobre derechos y deberes ciudadanos, que permanece intacto desde 1980, materializa los pilares jurídico-filosóficos del sistema educativo formal chileno. En él se define que la educación deja de ser una función primordial del Estado, y que son los padres quienes "tienen el derecho preferente y el deber de educar a sus hijos", correspondiendo al Estado "otorgar especial protección al ejercicio de este derecho" (art. 19, 10 ${ }^{\circ}$ ). Se establece, como derecho, la libertad de enseñanza, entendida como "el derecho de abrir, organizar y mantener establecimientos educacionales", y la potestad de los padres de escoger el establecimiento donde educar a sus hijos $\left(\operatorname{art.} 19,11^{\circ}\right)$. Se estipulan cuatro derechos de propiedad económica: "derecho a desarrollar cualquier actividad económica" (art. 19, n. 21), "derecho a no ser discriminado por el Estado y sus organismos en materia económica" (art. 19, n. 22), "derecho a la libertad para adquirir el dominio de toda clase de bienes" (art. 19, n. 23) y el "derecho de propiedad en sus diversas especies sobre toda clase de bienes corporales e incorporales" (art. 19, n. 24). No existen derechos colectivos, ni derecho a la participación.

Finalmente, para dejar claro el orden de prelación de los derechos, se establece un mecanismo de recurso de protección sólo para algunos derechos constitucionales. El derecho a la educación no cuenta con esta garantía, sí la tienen la "libertad de enseñanza" y los derechos de propiedad mencionados (art. 20).

\section{Institucionalidad y gestión del sistema escolar: municipalización de la edu-} cación pública y nuevo rol de los "sostenedores" privados en la educación

En el año 1981, se inicia un proceso de descentralización y privatización de la educación. Las escuelas estatales, que estaban directamente gestionadas por el nivel central del Ministerio de Educación (en la medida que no existen en Chile Estados 
federados), pasan a depender directamente de estructuras intermedias, los "sostenedores" municipales. Se favorece, además, el que sostenedores privados abran escuelas con los mismos subsidios estatales que reciben las escuelas municipales. Los sostenedores administran estos subsidios y deciden respecto de aspectos centrales de la vida escolar, como los planes anuales de cada escuela, la designación de directores, la reubicación de profesores, entre otros. En este modelo el Ministerio de Educación no tiene ingerencia alguna sobre el funcionamiento de las escuelas privadas (pagadas por los padres y apoderados) ni sobre las privadas subvencionadas (de administración privada, pero que reciben recursos del Estado) y, en la práctica, pierde gran parte de su capacidad de influir en la marcha de las escuelas municipales. El rol del Estado se reduce a financiar la enseñanza básica ( $1^{\circ}$ a $8^{\circ}$ grado) y media ( $9^{\circ}$ a $12^{\circ}$ grado), que cubre alrededor del $93 \%$ de los estudiantes, a controlar, formalmente, el cumplimiento de los requisitos que tienen los sostenedores para acceder a la subvención, a medir el rendimiento escolar y a orientar y supervisar los aspectos técnicopedagógicos. La "comunidad educativa", los actores reales, es espectadora en esta gestión, o la padece directamente a través de la represión escolar o del abuso de los derechos laborales de los trabajadores de la educación (docentes y asistentes de educación), ya que el profesorado pierde el carácter de funcionario público y su régimen jurídico queda definido por las leyes laborales del conjunto de los trabajadores del país, lo que implicó absoluta flexibilidad laboral, baja sustantiva de sus salarios y de sus condiciones de trabajo, deteriorándose fuertemente la profesión docente.

\section{El sistema de financiamiento educativo: subsidios portables a los alumnos/ as y privatización del gasto público}

El modelo recién descrito opera con un mecanismo de financiamiento a la demanda que consiste en un "sistema de subvenciones donde el Estado subsidia colegios privados esencialmente en el mismo nivel que los establecimientos municipales" (OCDE, 2004, p. 177), con la particularidad que los sostenedores privados no están obligados a rendir cuenta sobre dichos recursos, ni tienen regulación alguna para su uso. Este sistema de subsidios, llamados subvención escolar, son vouchers o subsidios portables. A través de ellos se financia a las escuelas y, por tanto, a los sostenedores, por medio de una subvención calculada por promedio de asistencia de los alumnos. Tiene por objetivo explícito introducir mecanismos de competencia entre los colegios: los establecimientos educacionales competirían por captar alumnos/as para recibir la subvención estatal y ello generaría una dinámica de mejora de los resultados académicos.

En esta lógica de competencia en el mercado, se crea un Sistema Nacional de Medición de la Calidad de la Educación (SIMCE), destinado a realizar mediciones de rendimiento de los alumnos en todos los establecimientos del país, cuyos resultados 
deben ser puestos en un ranking público a través de los medios de comunicación, de modo de entregar a los padres indicadores para contar con información para elegir el establecimiento donde educar a sus hijos.

Las transformaciones descritas modificaron la lógica del sistema y el sentido de la educación. Al término de la dictadura se había consolidado un sistema educativo mixto, financiado por el Estado, que alcanzaba una cobertura de más del 90\%, evidenciaba serios problemas de calidad y equidad, cubriendo la educación municipal más del $70 \%$ de la matrícula y la privada subvencionada casi el $30 \%$.

\section{La privatización del sistema de educación superior}

También a partir de los años ' 80 se desestructura el sistema de educación superior. Por una parte se descentralizan y precarizan las universidades estatales nacionales, creando a partir de ellas universidades estatales regionales con escaso financiamiento y, por otra, se abre el mercado para que privados abran sin mayores restricciones sus propias universidades e institutos de educación superior, dándoles plena autonomía. Lo que ha desembocado en una desregulación absoluta de la formación profesional, sin relación alguna con los movimientos del mercado del trabajo. Lo más grave es que se termina con la gratuidad de la educación superior, propiciándose el autofinanciamiento tanto de las universidades públicas como privadas, ya que se disminuye en un $50 \%$ el financiamiento a las universidades públicas. ${ }^{1}$ Además, los institutos profesionales, que dependían del Estado, son traspasados a los grupos empresariales de los distintos ámbitos de la producción, con lo cual se privatizan absolutamente (Inzunza et al., 2011). Esta nueva institucionalidad de la educación superior afectó seriamente también la formación docente, que quedó librada al mercado. La formación docente en servicio o perfeccionamiento sufrió el mismo proceso privatizador.

\section{Los ejes de la reforma educativa de los años ‘90}

Los grandes propósitos de la reforma educativa de los años '90, impulsadas por los gobiernos de la Concertación, ${ }^{2}$ fueron justamente la mejoría de la calidad y la equidad de los aprendizajes (Ministerio de Educación, 1998). Para ello se implementaron cinco grandes líneas de políticas educativas:

a) Aumento del gasto público en educación, el que se triplicó entre 1990 y 2004, alcanzando la cifra de 3000 millones de dólares (Cox, 2004) y ha continuado aumentando hasta la fecha. Un alto porcentaje de este gasto público se transfiere al sector privado vía subvenciones e inversiones en infraestructura.

b) Reforma curricular, a partir de la cual se renovaron los planes y programas correspondientes a todos los niveles de enseñanza básica y media del país. 
c) Mejoramiento de la profesión docente, con la promulgación de un estatuto que regula la carrera profesional y el marco laboral para los profesores del sector municipal y el establecimiento de incentivos y programas de perfeccionamiento para profesores.

d) Jornada escolar completa diurna, lo que ha implicado la construcción de una significativa cantidad de establecimientos escolares.

e) Articulación de programas en torno al mejoramiento de la equidad y la calidad de la educación, con la implementación, particularmente, de programas focalizados en los establecimientos que atienden a los sectores más populares.

Ninguna de estas políticas tocó los pilares estructurales del modelo diseñado en dictadura: municipalización, sistema de financiamiento por subvención, la libertad de los sostenedores privados para abrir establecimientos con financiamiento del Estado.

$\mathrm{Al}$ contrario, se avanza en medidas privatizadoras. La más grave de ellas fue la promulgación de la Ley de Financiamiento Compartido, que permite que el sector privado subvencionado por el Estado y los establecimientos municipales de educación secundaria puedan cobrar arancel a la familia, bajo el argumento que aquellas que estuvieran en condiciones de pagar podrían aportar al financiamiento de la educación, con el objetivo que el Estado ahorrase recursos para destinarlos a aquellos sectores con mayores necesidades. Sin embargo, en los hechos, el Estado siguió entregando prácticamente la misma cantidad de recursos a los establecimientos con financiamiento compartido y a los que no lo tenían. Ello significó un nuevo estímulo económico para el sector privado (fueron muy pocos los establecimientos secundarios municipales los que se suscribieron a esta modalidad de financiamiento). Un nuevo campo para la educación privada se abre con la Ley de Jornada Escolar Completa, la que, por primera vez en la historia del país, entrega recursos públicos a entes privados para la construcción de establecimientos educacionales.

\section{Los ejes de la reforma educativa de los ' 2000}

Luego de una década de aplicadas las medidas reseñadas, la evaluación de dichas políticas indicó que ni los mayores recursos materiales y económicos, ni los programas de focalización y sus planes de mejoramiento habían logrado resolver los problemas de calidad y equidad. Se observó, a partir de los resultados en pruebas estandarizadas de aprendizaje (SIMCE, PISA y TIMSs), que éstos subsistían (Rasczinski \& Muñoz, 2007; Valenzuela et al. 2008). Desde estos diagnósticos, se plantea la necesidad de instalar ya no sólo mecanismos de rendición de cuentas del rendimiento escolar, sino también del quehacer y de la gestión de los distintos actores, estableciéndose nuevos sistemas de evaluación que empiezan a aplicarse 
a partir del 2000. Se construye un Sistema de Aseguramiento de la Calidad de la Gestión Escolar, a nivel institucional; se inician procesos de acreditación de instituciones escolares realizadas por entidades privadas; y se elaboran diferentes marcos de actuación a nivel de actores específicos del sistema escolar (Docencia, mayo 2007).

Se empezaron, también, a cuestionar los mecanismos de financiamiento, planteándose que obstaculizaban el proceso de mejora vía competencia, dado que no entregaban los recursos adecuados según nivel socioeconómico de los estudiantes (Bellei, 2007; Donoso, 2006; González, 2000), y que además, el modelo de subvención, de valor similar por alumno, promovía que los establecimientos seleccionaran a los alumnos menos costosos de educar (Elacqua et al., 2009; Sapelli y Torche, 2002; Mizala y Romaguera, 2002).

Para resolver estos problemas, el año 2006, nuevamente sin alterar los pilares del sistema, se inicia la discusión de la Ley de Subvención Escolar Preferencial (SEP), que rige para la educación básica. Se trata de un ley paradigmática, pues articula diversos mecanismos de rendición de cuentas y de gestión anteriores a su promulgación, y al mismo tiempo define las próximas medidas de políticas educativas, las cuales pretenden modernizar el sistema para lograr seguir con el funcionamiento del mismo sin modificar sus bases.

Se trata de una ley de financiamiento que entrega recursos adicionales para disminuir la brecha de inversión por alumno (Larrañaga y Peirano, 2007; Elacqua et al., 2009) y estipula una política de gestión que busca establecer procesos de mejora continua de la gestión de las escuelas y de sus resultados (Raczynski y Muñoz, 2007; Cornejo y Redondo, 2007). De hecho, es una política que se inscribe en la generación, a nivel internacional, de nuevos mecanismos de gestión: procedimientos de acreditación de escuelas, incentivos al desempeño en la gestión, instalación de esquemas de asistencia técnica focalizada, exigencias de accountability (rendición de cuentas) y benchmarking (evaluación y comparación de prácticas). Ésta se sustenta en cinco pilares: 1) entrega de un monto extra de subvención por alumno "prioritario" (alumno determinado como vulnerable o pobre), que se concretiza con la firma de un "Convenio de Igualdad de Oportunidades y Excelencia Educativa", el que compromete al sostenedor con un conjunto de exigencias; 2) condicionalidad en la entrega de esta mayor subvención de acuerdo a los resultados SIMCE; 3) clasificación de todas las escuelas básicas, en las categorías "Autónoma", "Emergente", "En recuperación", a partir de lo cual se definen para ellas grados de mayor o menor autonomía para la utilización de recursos, gestión administrativa y pedagógica; 4) sistemas de supervisión y apoyo técnico pedagógico a las escuelas, ejecutados por agencias privadas; 5) establecimiento de sanciones por incumplimiento de las metas suscritas en los convenios, que van desde las multas hasta el 
cierre de los establecimientos. Todas estas medidas se empezaron a implementar gradualmente desde la promulgación de la ley el 2008.

El mismo año 2006, mientras se discutía esta ley, como consecuencia de un estallido social encabezado por los estudiantes, se evidenciaron, ante el conjunto de la opinión pública, los graves problemas de calidad y equidad del sistema educativo, por lo que el gobierno de Michelle Bachelet se vio obligado a alterar su programa de gobierno en materia educacional, abriendo camino a un debate que supuestamente conduciría a la concreción de una nueva ley de educación, que cambiaría algunos aspectos estructurales relevantes heredados de la dictadura. Efectivamente, en 2009, se promulga la Ley General de Educación (LGE), que reemplaza la Ley Orgánica Constitucional de Educación de 1980. Sin embargo, en lugar de recoger en ella las principales exigencias del mundo social (desmunicipalización, nueva institucionalidad y cambios radicales en el sistema de financiamiento), ésta fue construida por un acuerdo político cupular entre los partidos de gobierno y los de derecha, que no dio paso a ninguna reforma estructural. En síntesis, la LGE sigue supeditando el derecho a la educación a la libertad de enseñanza y de empresa, y consagra el lucro y la selección. Establece, además, la creación de una Agencia de Calidad (proyecto de ley que está aún en trámite en el Parlamento), que crea una superintendencia y servicios educativos descentralizados, acentúa los mecanismos de control y presión por resultados, consolida la exigencia de cumplimiento de estándares y la categorización de escuelas, reafirmando las políticas que habían empezado a cursar con carácter voluntario, para convertirlas en exigencias obligatorias para el sistema.

En algunas palabras, en esta ley se articulan todas las normativas de estándares de los marcos regulatorios de actuación de los actores - profesores, directivos, supervisores -, así como los mecanismos de la ley SEP para el conjunto del sistema escolar. Además, fortalece y amplía el espacio de acción para que agentes privados no sólo lucren con dineros del Estado, sino que controlen ideológica, técnica y pedagógicamente a escuelas y liceos.

El nuevo gobierno de derecha, que eligió al presidente Sebastián Piñera en enero 2011 y que empieza a gobernar en marzo del mismo año, ha decidido aplicar políticas de continuidad del consenso construido el año 2007, señalando que su prioridad es la aprobación de este proyecto de ley. Ha promulgado, además, una nueva ley - "Ley de Calidad y Equidad de la Educación" - en enero de este año 2011, en acuerdo nuevamente con gran parte de los senadores y diputados de la Concertación, que entrega mayor poder y recursos a los sostenedores municipales para administrar los establecimientos, lo que consolida la municipalización de la educación, al mismo tiempo que permite la flexibilización absoluta del Estatuto Docente, precarizando la situación laboral del profesorado que trabaja en este sector. 


\section{Resultados de la aplicación del modelo de mercado educativo}

Las implicancias de la aplicación de este modelo ideológico de educación, que ha extremado la privatización de nuestro sistema educativo, han sido múltiples y de amplias dimensiones. En este artículo destacaremos tres grandes tendencias que ayudan a tener una visión de conjunto de estos efectos reales: alta segmentación socioeducativa, crisis de pertinencia y sentido de la educación chilena, cambio de la cultura escolar y del rol docente.

\section{Alta segmentación socioeducativa}

Chile se encuentra ante un verdadero apartheid educativo, fenómeno absolutamente nuevo en la historia de este país. La educación gratuita, que hasta el año 1993 atendía más del 90\% de la matrícula total, a partir de la ley de financiamiento compartido, hoy apenas alcanza un $60 \%$, mientras los otros $40 \%$ se distribuyen de acuerdo a la capacidad económica de las familias, lo que provoca gran segmentación y segregación en el sistema escolar, donde cada estudiante se educa con sus pares desde el punto de vista social y económico. El extremo puede graficarse en que más del $80 \%$ de los niños de los quintiles más bajos de ingreso (Quintil I y II) estudian en escuelas municipales, mientras el $93 \%$ de los niños del quintil más alto de ingreso estudian en la educación privada pagada.

Además, en el sistema educacional existen inmensas distancias en los recursos educativos y económicos que reciben escuelas privadas, privadas subvencionadas y municipales, y enormes diferencias en los resultados de aprendizaje, entre niños de sectores socioeconómicos de bajos ingresos y de altos ingresos (OCDE, 2004; Redondo et al. 2004; Cornejo, 2006). A su vez, el propio sistema municipal, que recibe un financiamiento absolutamente insuficiente por parte del Estado para atender las complejas necesidades educativas de sus estudiantes, está también segmentado y fragmentado, pues depende de las capacidades financieras, técnicas y de gestión de cada comuna, que son disímiles, aunque en general son muy escasas. En Chile existen 345 comunas (por lo tanto 345 sostenedores municipales), sin embargo estimaciones recientes plantean que solo 120 de estos municipios (un tercio) poseen "un equipo profesional estable de apoyo" que cuenta, al menos, con un encargado de técnico pedagógico y un especialista en educación. Dentro de estos 120 municipios, sólo 32 tienen equipos técnicos de apoyo de mayor desarrollo y complejidad (obviamente estos municipios suelen ser los que manejan mayor presupuesto) (Montt, 2005). De este modo, la municipalización, más que un proceso de descentralización, ha sido un proceso de desresponsabilización y desentendimiento de la educación pública por parte del Estado chileno.

A este cuadro de segmentación, se suma el que los sostenedores privados, y también los municipales con alta demanda, seleccionan a los estudiantes a quienes 
quieren educar, y terminan eligiendo, en clave económica, a los que implican menos gastos (buenos rendimientos, conductas tranquilas, mucha presencia familiar, entre otros). De este modo, el sistema educativo termina, en los hechos, favoreciendo a quienes tienen más y no a quienes más lo necesitan. Así, la trayectoria educativa laboral de la mayoría de los estudiantes es "predecible" a muy temprana edad. Se consagra lo que la oCDE (2004) define como un sistema educativo "conscientemente estructurado por clases sociales", donde cada familia accede a distintos tipos de educación de acuerdo a su capacidad de pago.

Junto a ello se consolida un modelo de mercado que arrincona cada vez más a la educación pública, lo que genera un aumento explosivo de la privada: en 1980 el $7 \%$ de los alumnos/as de enseñanza básica y media asistían a establecimientos privados subvencionados, al año 2010 lo hacía más de un 50\%.

La ley de subvención escolar preferencial, que como señalábamos se orientaba en sus principios a resolver los problemas de segmentación y fragmentación, en los hechos lo que está haciendo es profundizarlos, al categorizar a las escuelas, estigmatizarlas y abrir la posibilidad de cerrar establecimientos si no logran subir sus rendimientos medidos por las pruebas estandarizadas. Por otra parte, avanza fuertemente en la privatización del sistema, al dejar a las escuelas sujetas a la supervisión de entidades privadas, donde priman, muchas veces, sus propios intereses empresariales y/ o ideológicos por sobre las reales necesidades de los establecimientos. Sin duda, todo ello se acentuará de aprobarse las nuevas leyes que siguen con esta misma lógica.

\section{Crisis de pertinencia y sentido de la educación}

Lo que venimos señalando explica por qué en Chile se suele centrar el debate sobre calidad educativa en torno a los resultados en pruebas estandarizadas de aprendizaje, perdiendo su pertinencia e imposibilitando la construcción de proyectos curriculares desde la comunidad. Por otra parte, el desempeño de Chile en estas pruebas es disímil. Si bien el país ha mejorado su ubicación relativa en pruebas como PISA, según los mismos expertos de PISA, los resultados más dependen del origen socioeconómico familiar de los estudiantes. Es por ello que los actores educativos (docentes, estudiantes, apoderados) sienten que ir a la escuela es someterse a una cotidianeidad escolar ingrata (Becerra, 2005; Cornejo, 2009), más aún cuando en general los procesos de participación han sido casi inexistentes, ya sea porque se prohíben legalmente o porque los actores están capturados por la sobrecarga de trabajo.

Lo que ha sucedido es que el concepto de calidad educativa pasó a ser colonizado por la lógica de un currículum nacional y de estándares (y mediciones) de aprendizaje. Los resultados de estas pruebas estandarizadas se hacen sinónimo de calidad, significando una mirada estrecha de asumir este concepto, asimilándolo sólo 
como buen rendimiento en determinadas asignaturas del currículum, sin incorporar todos aquellos otros ámbitos de este último y del desarrollo humano que debieran considerarse como atributos fundamentales de una educación integral de calidad. Estas pruebas censales van adquiriendo cada vez mayor relevancia, constituyéndose, en la práctica, en el eje articulador de todas las políticas que en definitiva se orientan a mejorar la "productividad" (elevar los puntajes en las pruebas SIMCE) (Assaél, 2011).

Piia Seppänen, investigadora del Centre for Research on Lifelong Learning and Education, de Finlandia, en entrevista ofrecida al periódico El Mercurio el 29 de noviembre de 2010, cuestionó la función de las continuas evaluaciones que se aplican en Chile, planteando que lo que se está produciendo en nuestro sistema de educación, con los mecanismos de evaluación estandarizada y los mecanismos de incentivo a los mejores rendimientos, la educación pública abandona uno de sus objetivos fundamentales que es la formación de ciudadanos comprometidos con su país, con la democracia como sistema de resolución de conflictos, con la valoración de la comunidad y su entorno. Agrega que estos mecanismos de evaluación constantes no sólo deterioran los contextos en que se hace la educación, sino también los vínculos interpersonales.

En efecto, lo que se ha evidenciado durante todos estos años es que los resultados de las pruebas estandarizadas están estrechamente ligadas al nivel socioeconómico de los alumnos y, por tanto, son los establecimientos municipales que atienden a los sectores populares, y que mayoritariamente no discriminan, los que obtienen peores puntajes, en cambio las escuelas privadas subvencionadas, que sí seleccionan, aparecen mejor ubicadas en los ranking, lo que ha llevado a estigmatizar a las primeras y a sostener la idea que la educación pública es mala. Ello se refleja, por ejemplo, en que al año 2009, menos del 39\% de los estudiantes de primero básico asistían a una escuela municipal. Así, la escuela pública termina transformándose en el espacio educativo para aquellos pobres que no convienen, ni pueden acceder, como clientes, al sector privado. Se niega, de esto modo, el carácter sustantivo de cualquier democracia: la escuela pública como el ámbito en el que se pueden compartir distintas realidades (sociales, culturales, ideológicas, étnicas).

\section{Cambio de la cultura escolar y del rol docente}

La presión ejercida a través de las metas de resultados en pruebas de aprendizaje estandarizadas conduce a que en los establecimientos educacionales se tienda a enseñar lo que se evalúa, reduciéndose el curriculum fundamentalmente a lenguaje, matemáticas y ciencias, y a los aprendizajes que dentro de estas disciplinas pueden ser evaluados de manera estandarizada. Las prácticas pedagógicas tienden a centrarse exclusivamente en el entrenamiento de estas pruebas estandarizadas, en abierto menoscabo de asignaturas como filosofía, arte, educación física, que cada vez tienen 
menos espacio en el curriculum, así como de procesos de enseñanza y aprendizaje que favorezcan el desarrollo de sujetos con una formación más integral, reflexivos y críticos.

Por otra parte, los mecanismos de presión, control y rendición de cuentas han afectado seriamente la autonomía profesional, provocando desmoralización en el ejercicio docente, así como elevados niveles de angustia y stress, en un sistema que los obliga a centrarse en la enseñanza de los resultados medibles en desmedro de otras áreas que ellos consideran tanto o más importantes, como lo son la atención a problemas psicosociales o emocionales, que viven la gran mayoría de los niños y jóvenes, particularmente en los sectores populares (Assaél, 2011; Cornejo, 2009). Estas políticas de accountability ubicarían a los docentes en una posición "híbrida", en la que no saben si deben educar o enseñar para los exámenes (Darling-Hammond, 2004, en Contreras y Corbalán, 2010). Así, comienzan a construir "simulaciones pedagógicas", planificando y calculando acciones con el fin de satisfacer las demandas externas, ser evaluados positivamente y responder al creciente flujo de vigilancia al cual están expuestos, pero en lo sustantivo no modifican sus prácticas pedagógicas más allá de lo requerido por la evaluación (Ball, 2003; Case, Case \& Catling, 2000; Taylor Webb, 2006, en Contreras y Corbalán, 2010).

Ello se acrecienta al desresponsabilizarse el Estado de los resultados que tiene el sistema educativo, entregando dicha responsabilidad a los actores, culpabilizando a las escuelas, a los padres, a los profesores, y a los propios estudiantes, de los resultados de aprendizaje (Assaél, 2011). La rendición de cuentas estaría operando como un dispositivo de vigilancia que enseña a cumplir y presionar a otros para el cumplimiento de la tarea, lo que se logra visibilizando e individualizando las supuestas deficiencias, que son fácilmente medidas a través de los resultados de las pruebas de los alumnos y de la supervisión de las prácticas de planificación y ejercicio de la docencia en aula (Lipman, 2009).

Se instalan, así, más que relaciones de colaboración, una cultura de competencia, no sólo entre docentes por obtener incentivos económicos ligados a resultados de pruebas estandarizadas y de desempeño, sino también entre estudiantes y padres y apoderados; cada uno asume como un problema individual su éxito o fracaso. Desde esta óptica, la identidad profesional empieza a redefinirse a partir de los principios de la nueva gestión pública, lo que implicaría la entrada de un nuevo conjunto de valores y un nuevo comportamiento moral, más cercano a una ética empresarial que al tradicional ethos del profesor (Gewirtz y Ball, 2000; Whitty, Power y Halpin, 1999, en Contreras y Corbalán, 2010).

Esto empieza a pesar en los propios estudiantes, que se percatan de la lógica empresarial y el modo en que está alterando las pautas de socialización de las 
escuelas. La educación empieza a perder sentido y significancia para ellos, lo que explica, en parte, las movilizaciones estudiantiles del año 2006 contra un sistema que les enseñaba a ser trabajadores sumisos, aún antes de cualquier evaluación o esfuerzo colectivo (ni qué decir individual). De esta lucha emergió una ética mínima construida en oposición a los valores que 30 años de imposición de un sistema de competencia habían minado. La crisis de sociabilidad manifestada en una especie de juventud que parecía "no estar ni ahí con nada" y a la que el modelo se le adecuaba en sus formas de valorización individual, reventó, iniciándose, como veremos a continuación, un proceso de búsqueda de construcciones colectivas.

\section{El movimiento por la educación y sus propuestas}

La participación de los actores sociales en la educación pública tiene larga tradición en Chile. A partir de las primeras iniciativas educativas autónomas impulsadas por las sociedades de socorros mutuos a finales del siglo xIX, se pueden señalar numerosas experiencias de participación del movimiento social en las políticas de educación en la historia de nuestro país. A principios del siglo xx son los profesores quienes comienzan a intervenir en el debate público, con iniciativas como el Congreso Nacional de Educación Secundaria del año 1912, o con su decisiva participación en el proceso de movilización social que constituyó la Asamblea Obrera de Alimentación Nacional en el año 1919 y los movimientos docentes que se desarrollaron en la siguiente década. Paralelamente, se constituían experiencias como las escuelas federales racionalistas, sostenidas por el movimiento obrero, antecedente importantísimo para una educación pública entendida como una educación con participación de la comunidad (Reyes, 2009).

Este movimiento de transición de siglo representa el origen de la propuesta educativa del movimiento popular en Chile, la que en oposición a la mirada del Estado nacional-mercantil, pone a la comunidad y a la persona al centro de la labor educativa. No son los imperativos del Estado, ni los del mercado, el fin único de la escuela pública; el interés de la comunidad local, las problemáticas cotidianas o un proyecto emancipador hacen parte de las propuestas del mundo popular (Reyes, 2005).

A mediados del siglo xx, comienza la resistencia al hegemónico patrón organizador de la vida y la conciencia. Los estudiantes comienzan a expresar su descontento en las escuelas y en las calles, el reclamo por el pase escolar antecede a la demanda de participación, que, con el correr de los años, irá incorporando el movimiento juvenil escolarizado. En síntesis, trabajadores de la educación, estudiantes secundarios y universitarios, entre otros actores sociales, han estado presentes en la historia del sistema educativo, poniendo siempre en tensión la libre instalación de proyectos hegemónicos ajenos a los intereses mayoritarios (ОРЕCH, 2010a). 
El movimiento de estudiantes secundarios, que irrumpe públicamente durante el año 2006, como lo vimos en el acápite anterior, es ejemplo de este devenir histórico (González et al., 2006). Esta movilización contó con un nivel de masividad y apoyo ciudadano no visto, desde la entrega del Poder Ejecutivo por parte de Pinochet. En términos generales, podemos decir que este movimiento se

\begin{abstract}
(...) organizó a partir de la articulación de la Asamblea Coordinadora de Estudiantes Secundarios (ACES), instancia surgida desde los centros de alumnos y colectivos estudiantiles organizados principalmente en los colegios municipales del centro de la capital que atienden alumnos de elite económica e intelectual, pues seleccionan a sus alumnos y en algunos otros colegios municipales y particulares subvencionados por el Estado, ubicados en el mismo sector. (González et al., 2006, p. 3)
\end{abstract}

A los pocos días de iniciados los paros y las ocupaciones de los establecimientos de educación secundaria de Santiago, el movimiento genera una explosiva adhesión que, para sorpresa de muchos, sumó rápidamente a los estudiantes y profesores de los liceos y escuelas de las zonas periféricas y otras regiones del país. La ACES adquiere un carácter nacional. Se desencadena un proceso generalizado de tomas y paros, testimonio del acercamiento entre los jóvenes más marginados de la sociedad chilena (de los llamados liceos periféricos o urbano-populares) y los jóvenes de las clases medias y populares con posibilidades de "integración social". Este fenómeno de unidad, inédito en el Chile de post dictadura, caracteriza la radicalidad, en el sentido de masividad social, de este movimiento. Durante los paros nacionales de los días 30 de mayo y 5 de junio de 2006, se calcula que hubo más de un millón de estudiantes secundarios movilizados (de un total aproximado de un millón doscientos mil estudiantes en el país). A esta cifra hay que sumar el apoyo de estudiantes de los cursos superiores de educación básica, del Colegio de Profesores, de asociaciones de padres y apoderados y de la Confederación Nacional de Estudiantes Universitarios. Ningún movimiento social de post dictadura había mostrado los niveles de masividad y convocatoria social con los que contó este movimiento. No es aventurado afirmar que la movilización de los estudiantes y las tomas pacíficas de los liceos configuran un nuevo espacio público (de intereses en común) que representa el "sufrimiento colectivo" y el desamparo social que experimentan vastos sectores de la población chilena en el actual modelo de desarrollo neoliberal (González et al., 2006).

De este modo, tras la desarticulación del tejido social y la disolución de la conciencia de "lo público", promovida por la dictadura y consolidada en los gobiernos democráticos, el movimiento social por la educación pública comienza a remontar su camino y a recuperar en su conciencia y en sus propuestas una escuela para todos. Así, a las sucesivas movilizaciones estudiantiles, han comenzado a sumarse otros actores educativos que discuten acerca de qué implica, hoy, construir una educación pública integral al servicio de las mayorías y no de los intereses de las elites. 
$\mathrm{Al}$ calor de la movilización de estudiantes, padres y trabajadores de las escuelas, un nuevo significado de la educación comienza a reconstituirse en oposición al modelo competitivo e individualizante que propone el mercado. El imperativo que el Estado se haga cargo de sus escuelas, un mejor financiamiento a las escuelas empobrecidas y la mayor participación de la comunidad en la toma de decisiones empiezan a consolidarse en las demandas y propuestas de los actores educativos, en contraposición a la iniciativa empresarial obsesionada con la competencia entre escuelas y los estándares (ОРеCH, 2010b).

Durante los últimos años, los actores sociales han elaborado una serie de propuestas que, poco a poco, van constituyendo un articulado de puntos de encuentro tras los cuales se comienza a organizar la demanda educativa actual, orientada a la elaboración y construcción de un proyecto educativo que fortalezca la democracia, promueva las comunidades locales, apoyado decididamente por el Estado, no discriminatorio, pertinente al desarrollo de cada persona, integral, que asegure la libertad de los ciudadanos.

\section{Notas}

1. Hasta 1981 en Chile existían dos universidades públicas y seis privadas, de las cuales tres eran católicas y tres laicas; todas las cuales surgieron de iniciativas particulares pero en estrecha relación con el Estado, pues si bien eran autónomas contaban con financiamiento estatal. Hoy existen alrededor de cien universidades privadas.

2. La Concertación es la coalición de partidos políticos de centro izquierda que gobernó el país desde 1990-2010, integrada por los partidos Demócrata Cristiano, Socialista, Radical/Social Demócrata y Por la Democracia.

\section{Referencias}

ASSAÉL, J. Políticas públicas en educación: el caso de Chile. In: Elichiry, N. (Comp.). Políticas y prácticas frente a la desigualdad educativa: tensiones entre focalización y universalización. Buenos Aires: Novedades Educativas, 2011. p. 81-102.

BECERRA, S. El clima educativo en establecimientos que han desarrollado esfuerzos de innovación, en el marco de la reforma educativa chilena; ponencia en 18. Encuentro de Investigadores en Educación. Santiago de Chile: CPEIP, 2005.

BELLEI, C. Expansión de la educación privada y mejoramiento de la educación en Chile: evaluación a partir de la evidencia. Revista Pensamiento Educativo, Santiago de Chile, v. 40, n. 1, p. 285, 2007.

CHILE. Ministério de Educación. Reforma educativa en marcha. Santiago de Chile: Ministerio de Educación, 1998. 
CONTRERAS, P.; CORBALÁN, F. ¿Qué podemos esperar de la Ley de Subvención Escolar Preferencial? Revista Docencia, Santiago de Chile, n. 41, p. 4-16, 2010.

CORNEJO, R. El experimento educativo chileno 20 años después. Revista REICE, Santiago de Chile, v. 4, n. 1, p. 118-129, 2006.

CORNEJO, R. Condiciones de trabajo y bienestar/malestar docente en profesores de enseñanza media de Santiago de Chile. Educación y Sociedad [online], Santiago de Chile, v. 30, n. 107, p. 409-426, 2009.

CORNEJO, R.; REDONDO, J. Variables y factores asociados al aprendizaje escolar: una discusión desde la investigación actual. Revista de Estudios Pedagógicos, Valdivia, v. 36, n. 2, p. 155-175, 2007.

COX, C. (Comp.). Políticas educacionales en el cambio de siglo: la reforma del sistema escolar de Chile. Santiago de Chile: Universitaria, 2004.

DONOSO, S. Financiamiento y gestión de la educación pública de los años 90. Cadernos de Pesquisa, Sao Paulo, v. 36, n. 127, p. 151-172, 2006.

ELACQUA, G. et al. La toma de decisiones de un sostenedor: análisis a partir de la Ley ser. Serie En foco Educación, Santiago de Chile, n. 1, 2009.

GESTIONAR, ¿Cómo? ¿Para qué? Revista Docencia, Santiago de Chile, n. 31, p. 39-53, 2007.

GONZÁLEZ, J. et al. "Estamos dando clases": significados y perspectivas del movimiento nacional de estudiantes en Chile. Boletín Referencias, Santiago de Chile, v. 3, n. 19, p. 2, jul. 2006.

GONZÁLEZ, P. Una revisión de la reforma del sistema escolar en Chile. Serie Economía, Santiago de Chile, n. 92, 2000.

INZUNZA, J. et al. Formación docente inicial y en servicio en Chile: tensiones de un modelo neoliberal. Revista Mexicana de Investigación Educativa, México, DC, v. 16, n. 48, p. 267-292, 2011.

LARRAÑAGA, O.; PEIRANO, C. Impacto distributivo de la subvención preferencial. Trabajos de investigación en políticas públicas, Departamento de Economía, Universidad de Chile, Santiago, 2007.

LIPMAN, P. Más allá de la rendición de cuentas: escuelas para nuevas formas de vida. Revista Docencia, Santiago de Chile, n. 38, p. 26-44, 2009.

MIZALA, A.; ROMAGUERA, P. Equity and educational performance. Economía, Washington, DC, v. 2, n. 2, p. 219-262, 2002. 
MONTT, P. Ponencia presentada en la ex sede del Congreso Nacional con ocasión de la entrega oficial del Informe ocDE a las autoridades nacionales. Santiago, 2005.

OBSERVATÓRIO CHILENO DE POLÍTICAS EDUCATIVAS (OPECH). De actores secundarios a estudiantes protagonistas 2.0. Santiago de Chile: Quimantú, 2010a.

OBSERVATÓRIO CHILENO DE POLÍTICAS EDUCATIVAS (OPECH). Alternativas y propuestas para la auto educación en Chile. Santiago de Chile: Quimantú, 2010b.

ORGANIZACIÓN PARA LA COOPERACIÓN Y EL DESARROLLO ECONOMICO (OCDE). Revisión de Políticas Nacionales de Educación: Chile. Paris: OCDE; Centro para la Cooperación con Países no Miembros, 2004.

RACZYNSKI, D.; MUÑOZ, G. Reforma educacional chilena: el difícil equilibrio entre la macro y la micro política. Santiago de Chile: cieplan, 2007.

REDONDO, J. et al. Equidad y calidad de la educación en Chile. Santiago: Universidad de Chile; Vice-Rectoría de Investigación; Facultad de Ciencias Sociales, 2004.

REYES, L. Movimientos de educadores y construcción de política educacional en Chile (1921-1932 y 1977-1994). 2005. Tesis (Doctorado en Historia) - Facultad de Filosofía y Humanidades, Universidad de Chile, Santiago de Chile.

REYES, L. Educando en tiempos de crisis: el movimiento de escuelas racionalistas de la federación obrera de Chile, 1921-1926. Cuadernos de Historia, Santiago de Chile, n. 31, p. 92-122, 2009.

SAPELLI, C.; TORCHE, A. Subsidios al alumno o a la escuela: efectos sobre la elección de colegios públicos. Cuadernos de Economía, Santiago de Chile, v. 39, n. 117, p. 175-202, 2002.

VALENZUELA, J.P. et al. Evolución de la segregación socioeconómica de los estudiantes chilenos y su relación con el financiamiento compartido. Santiago de Chile: FONIDE; Ministerio de Educación, 2008. (Informe final).

Recebido em março de 2011.

Aprovado em maio de 2011. 